\title{
Project-specific Process Configuration in Virtual Enterprises
}

\author{
C. Rupprecht and T. Rose \\ Research Institute for Applied Knowledge Processing (FAW), Germany \\ Em: \{Christian.Rupprecht IThomas.Rose\}@faw.uni-ulm.de \\ E. van Halm and A. Zwegers \\ Baan Development, Netherlands \\ Em: \{evhalm I azwegers\}@baan.nl
}

Keywords Process modelling, process configuration, project management, virtual enterprises

\begin{abstract}
Virtual enterprises have great difficulty in defining and adapting their business processes across the members of the virtual enterprise. We introduce an approach that allows (semi-)automatic (re-) configuration of one-of-a-kind business process models fitting the specific requirements and constraints of a project in a virtual enterprise. Our approach is based on the idea of making these requirements and constraints explicit by representing them in a model for each project.
\end{abstract}

\section{INTRODUCTION}

Virtual enterprises - temporary networks of enterprises that are formed for one-of-a-kind projects - have great difficulty in defining and adapting their business processes across the members of the virtual enterprise. Current practice is to use a rather general process model and to manually fill in the details from all members from scratch for each project. This practice is time consuming, costly, and error prone.

The business processes of a specific project in a virtual enterprise are influenced by many factors, such as customer requirements, legal constraints, and resources. We introduce an approach that allows (semi-) automatic (re-)configuration of one-of-a-kind business process models fitting the specific requirements and constraints of a project in a virtual 
enterprise. In this context, semi-automatic means generating proposals for adaptation going along a guided dialog with the user. In our approach, requirements and constraints will be made explicit and represented in a model. We address the definition and adaptation of business processes as a configuration problem.

The configured project-specific models will be transferred to multiple project management applications that control the execution of the defined business processes. Each member of the virtual enterprise will receive its part of the configured models in order to execute its business processes as specified in the model. Software support for our approach will significantly reduce costs and time-to-market and increase quality and responsiveness-tomarket in the project industry.

\section{PROCESS MODELLING IN VIRTUAL ENTERPRISES}

\subsection{Project Management}

Project management is about controlling cost, scope, and time. Activities during the initial stages of a project are scope management, cost engineering, and scheduling. Scope management is the process to define, change and monitor deliverables, the project structure, and the resources required. Scope management is needed before cost engineering and scheduling can start. Cost engineering has basically two phases, namely estimation and budgeting. Finally, scheduling is the process of determining the project lead-time. An activity network is made and the resource assignment(s) are scheduled according to their available capacity [5].

One of the characteristics of a project is its uniqueness. Projects are set up for specific customers, demanding specific results. Due to this uniqueness only a small part of the scope of all projects within an organisation can be standardised. Obviously, the extent of standardisation varies by organisation and type of projects. By means of standards, templates, and copying from previous projects, one can reuse existing (standard) information of deliverables, parts of the project structures, and/or required resources. Information could be for example:

- About deliverables: relationships, lead times, sales values

- About project structure: hierarchical relations, activity networks, durations, earned value methods

- About required resources: cost/sales prices, quantities, product configurations 
Nevertheless, a lot of time and effort is spent to define the parts that are not standard. For that part of the scope, one must maintain the remaining part of the estimate, budget and/or schedule. In order to reduce the steps in this process, reference models can be used. A reference model reflects all possibilities and is configured for the situation based on input variables.

\subsection{Process Modelling}

In virtual enterprises, processes need to be planned for each unique project. In this paper, we focus on the definition and adaptation of one-of-akind business processes, i.e. networks of activities. Before planning, coordination, and execution of the business processes of a virtual enterprise, they need to be defined, and during process execution they may have to be adapted due to new or changing requirements. This is particularly relevant for processes in the domain of complex system engineering $[2,4]$.

We define a process as a set of temporally or logically ordered activities intended to reach a goal involving resources. It can be regarded as a system where the elements are activities and resources and the relations are the sequential or logical dependencies between those elements. The set of relations describes the process structure.

A process model is a mental or explicit representation of original processes. In general, directed graphs are used for the explicit representation of processes. When we speak of process models in this paper, we mean semi-formal, computational representations in symbolic notation, i.e. general process elements like activities and their relations are represented by formal symbols (boxes and vectors) and additional information are attached nonformally, e.g. naming the symbols in natural language.

Process models capture know-how about ways of working in the past or intended in the future. However, a static process model contains no information about why work had or has to be done in a certain way. With our approach, we aim at creating generic, reusable representations of process configuration knowledge that can be applied across a variety of future process cases. The objective is to provide "guidance, suggestions, and reference material to facilitate human performance of the intended process" [1].

\subsection{State-of-the-art}

Current practice in virtual enterprises is to use a rather general process model and to manually fill in the details from all members from scratch for 
each project. This practice is time consuming, costly, and error prone. At the same time, global competition compels enterprises to drastically reduce their project duration in order to be competitive. For instance, to stay competitive in the power plant industry, an average project duration will have to be decreased from 24 months to 15 months in a near future.

Prevailing process models and supporting tools lack in the adaptation of actual processes. That is, there are sophisticated design, analysis, and management methods and tools, but methods for customising processes in the design and even execution phase are sparse. For example, one of the leading commercial process modelling tools, ARIS, allows to reuse functions that have been modelled and stored before. However, automated assistance for the context-specific deployment of such existing function descriptions is restricted to the matching of the function names. ARIS does not offer any support for the configuration of process models according to explicitly specified project requirements and constraints.

Some related research work on configuration and adaptation of process models and their restrictions are presented in [3].

\section{APPROACH}

\subsection{Core Idea}

Figure 1 shows the problem addressed in this paper. The business processes of a specific project are influenced by many factors, such as customer requirements, legal constraints, and resources. These requirements and constraints will be made explicit and represented in a model. A project manager has to take all these requirements and constraints into account when he/she initially performs project management activities, such as scope management, cost engineering, and scheduling. Likewise, all these factors have to be considered when process execution needs to be adapted to new circumstances. Nowadays, adaptation of business processes to specific project constraints and requirements is a manual, iterative, and difficult task. (Semi-)automatic configuration and adaptation will considerably ease this process. The configured project-specific models will be transferred to multiple project management applications that control the execution of the defined business processes. Each member of the virtual enterprise will receive its part of the configured models in order to execute its business processes as specified in the model. 


\subsection{Conceptual Framework}

Our research will result in a methodology and a prototype for process adaptation based on configuration. The main pieces of knowledge for the process configuration prototype are reuseable process building blocks, requirements and constraints, and configuration rules.

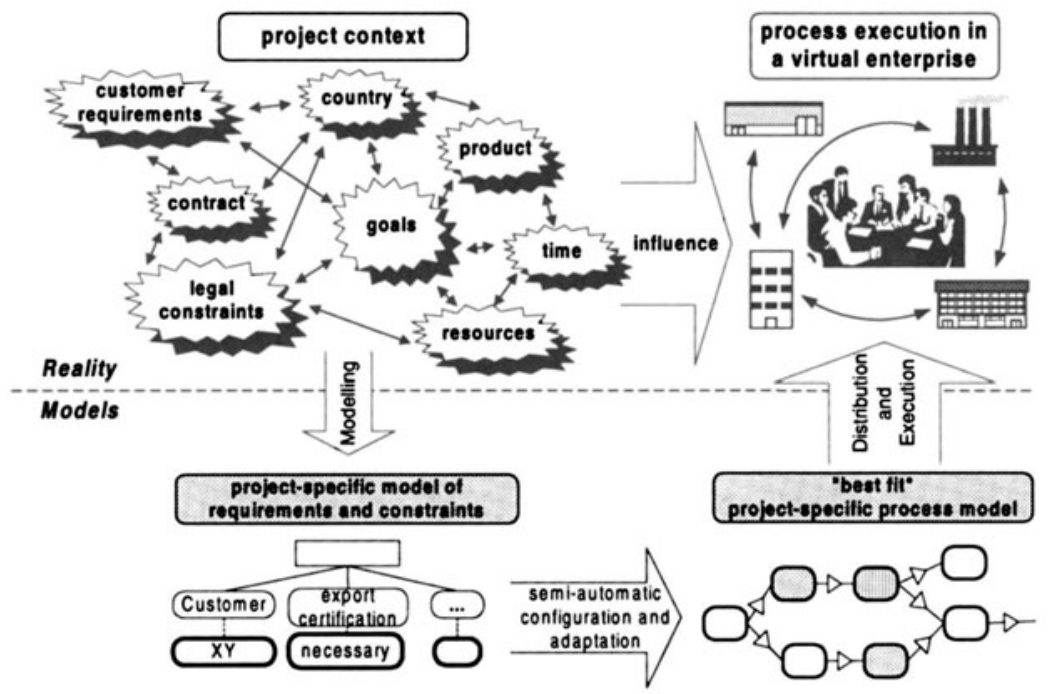

Figure 1 Core idea of process configuration and adaptation

For complex and innovative business processes, it is not sufficient to document a best-practice process once and follow this pattern forever in all future process cases. However, some smaller parts of a process are repetitive, i.e. for a certain task, the same activities are executed in the same sequence within different projects. For reasons of higher reusability and flexible connectivity, an extensive process model can be stripped down to temporally and logically isolated units called process building blocks. Such building blocks should have only few interfaces to other process models or building blocks, and they should "know" how they may be connected to other building blocks. In that case, a project-specific business process is created from combining such building blocks in unique combinations. In a virtual enterprise, each company keeps its set of process building blocks in a library.

Requirements and constraints have influence on the design of processes. They can be used to describe the project-specific context of a process and form the main input parameters to the configuration process. Options specify a requirement for a specific project, e.g. physical dimensions and expected 
due date of a deliverable. In order to use the set of requirements and their options in the configuration rules, the requirements can be derived from an existing information system whereas the options are entered at the moment of configuration. Taking into account dependencies between requirements, the configuration application also offers assistance for modelling the requirements in a specific project.

Experience in process modelling is broken down into single design decisions that are captured in terms of configuration rules. A configuration rule consists of a condition part which refers to constraints and an execution part which triggers configuration operators. Configuration rules are represented as dependencies among constraints and process building blocks. By applying these construction rules to a specific model of constraints and requirements, proposals for adaptations to the process model are generated. Together with the reuseable process building blocks, the set of configuration rules form generic reference process models. Generic means that the process models are automatically adaptable to a specific context via defined operators.

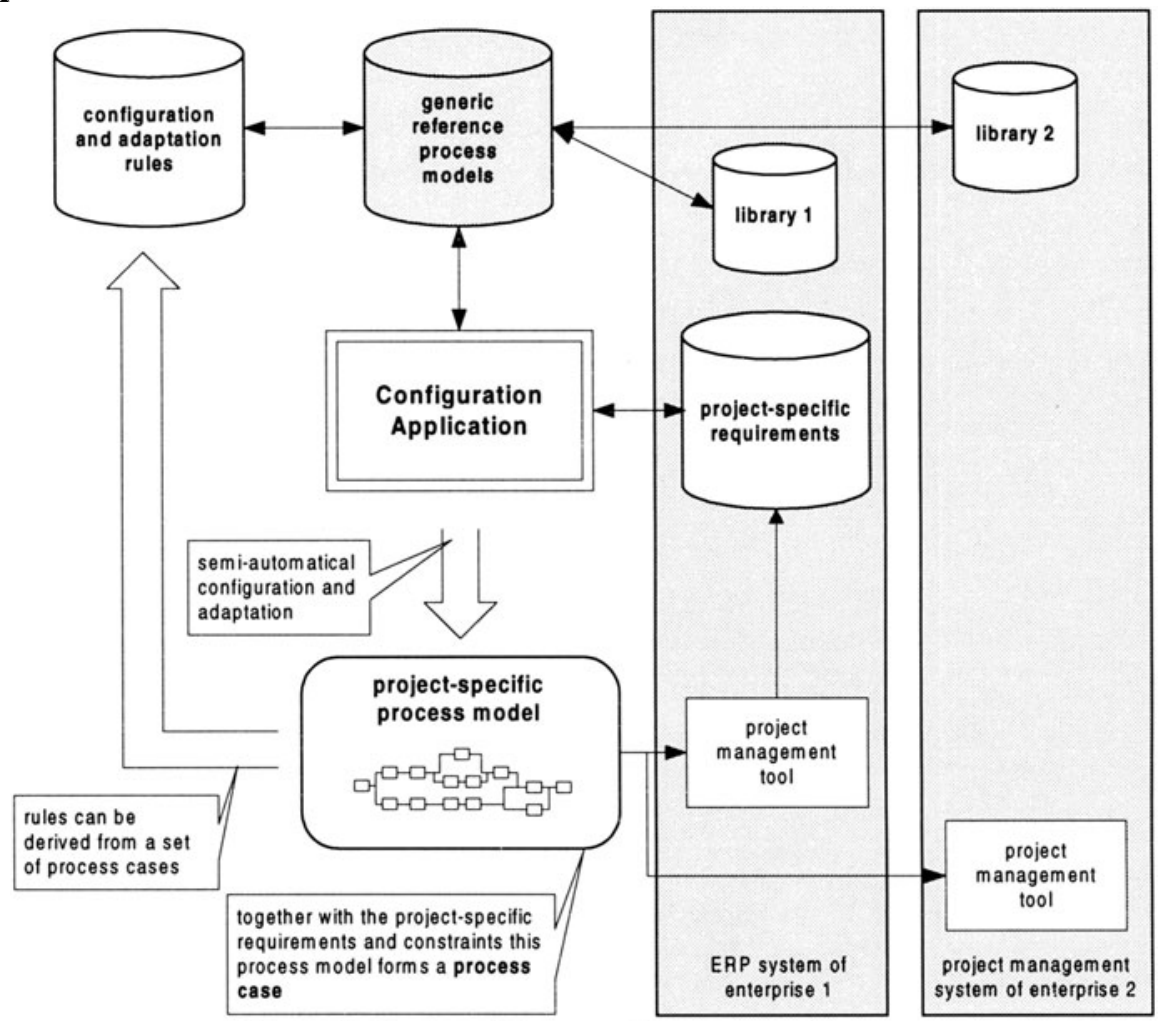

Figure 2 Basic architecture of a process configurator 
Figure 2 shows an initial architecture for the process configuration prototype. The prototype will consist of a front-end (Configuration Application) in which reference process models will be defined, and in which models of project-specific business processes can be configured. The backbone will provide necessary data stored in libraries, and it will contain the requirements which are input to the configuration. Business processes can be planned, co-ordinated, and executed with the help of project management tools based upon the configured process models.

Modelling the process relationships between the members of a virtual enterprise is a key element of the configuration application. This will be achieved by de-coupling the configuration application from any other information system, and by incorporating data from libraries belonging to different members of a virtual enterprise. The configuration application defines with which information system it has to interface in order to use the library data in the reference process models.

The knowledge base is extendable, i.e. users can define new constraints, requirements, reference process models, process building blocks, and construction rules at any time on a generic level and add them to the existing constraints, models, and rules. By collecting and maintaining such knowledge from different engineers, process modelling experience (i.e. the reasons for process design decisions in a specific context) can be captured and reused for future process modelling cases. A set of process cases can serve as a basis for computational analysis, in order to make the knowledge about dependencies explicit in terms of configuration rules.

\section{CONCLUSION}

Our research will deliver a methodology and prototype for process configuration and adaptation in virtual enterprises, mainly concentrated on project industries. The technological approach brings four main innovations:

- In a (semi-)automatic way, one-of-a-kind business processes will be configured. Configuration technology will be used to define specific business processes tailored to specific requirements;

- The specific business processes that are generated in the configuration application can be distributed to multiple participants of the virtual enterprise;

- Processes can be adapted during the entire project lifecycle;

- The configuration application is open in order to receive process and product architecture data from multiple information systems.

For industrial enterprises, there are a number of benefits. For example, responsiveness during the bid stage is increased. Making a bid to acquire a 
project requires a lot of effort. Knowledge is very often fragmented over different people and hardly formalised. With process configuration, it should be possible to capture some of the knowledge, thereby reducing the amount of work and the duration of bid preparation phases.

Another benefit is the increased efficiency in setting up projects. Nowadays, templates automate a lot of the work in setting up a project. A process configuration tool can bring this one step further, since it has the capability to be more specific about a situation and determine more details than a template ever can. With reconfiguration capabilities, the changes in a project can be executed without a lot of effort.

Process configuration also increases an organisation's ability to learn. It is very hard to learn from projects due to their uniqueness. Trying to find out what parts of different projects can be compared is quite difficult. If projects are generated from the same reference model, it will be easier to point out the comparable areas.

\section{REFERENCES}

[1] Curtis, B., M.I. Kellner, and J. Over. (1992). Process Modelling. Communications of the ACM, Vol. 35, No. 9, pp. 75-90.

[2] Negele, H., E. Fricke, L. Schrepfer, and N. Härtlein. (1999). Modelling of Integrated Product Development Processes. In: Proc. $9^{\text {th }}$ Annual Int. Symposium of INCOSE Systems Engineering: Sharing the Future. Brighton, UK.

[3] Rupprecht C., M. Fünffinger, H. Knublauch, and T. Rose. (2000): Capture and Dissemination of Experience about the Construction of Engineering Processes. In: Proceedings of the $12^{\text {th }}$ Conference on Advanced Information Systems Engineering (CaiSE*00). Stockholm, Sweden, pp. 294-308.

[4] Schott, H., A. Sieper, T. Rose, M. Fünffinger, C. Rupprecht, C. Schlick, and M. Mühlfelder. (2000). Process Knowledge Management in Concurrent Engineering. In: Proceedings of the $2^{\text {nd }}$ European Systems Engineering Conference (EuSEC 2000). Munich, Germany.

[5] Van Halm, E. (2000). Project Configuration - Summary of the Market Requirements. Internal Baan Development document. 